\title{
An Integrated Model of Semantic and Conceptual Interpretation from Dependency Structures
}

\author{
Udo Hahn Martin Romacker \\ Text Understanding Lab, [Gir] Group, \\ Freiburg University, D-79085 Freiburg, Germany \\ http://www.coling.uni-freiburg.de/
}

\begin{abstract}
We propose a two-layered model for computing semantic and conceptual interpretations from dependency structures. Abstract interpretation schemata generate scmantic interpretations of 'minimal' dependency subgraphs, whilo production rules whose specification is rooted in ontological categories derive a canonical conceptual interpretation from semantic interpretation structures. Configurational descriptions of dopendency graphs increase the linguistic gonerality of interpretation schomata, while interfacing schematia and productions to loxical and conceptual class hicrarchies reduces the anomit, and complexity of scmantic specitications.
\end{abstract}

\section{Introduction}

The syntax/semantics interface has always becn a matior of concern for constituoncy-based feature grammar theorics (cf., c.g., Croary and Pollard (1985), Moore (1989), Dallymple (1992), Wedckind and Kaplan (1993)). Within the dependency grammall community, far less aticution has been paid to this topic: As a conseguence, there is no consensus how syntactic dependency structures night be adequately transformed into somantic: interpetations (cf., Ia ajicova (1987), Milward (1992), J combardo et al. (1.998) for alternative proposals).

In this paper, we introduce a two-layered interpretation model. In a first pass, depondency graph stiructures which result from incromental parsing are immediatoly submitted to a scmantic interpretation process. Such a process is triggered by gencoal schemata whenever a semantically interpretable subgraph of a syntactic dependency graph becomes available (cf. Section 3). As a result, loxical items and the dependency relations holding betwoen them are directly mapped to associated conceptual entities and rolations at the level of semantic representation (cf. Sections 4 and 5). In a subsequent step, the (quasi-inferential) implications of the knowledge representation structures omerging from the semantic interpretation step are accounted for by a process we here rofer to as conceptual interpretation. The corresponding operations relate to the concep- tual representation level only and are triggered by a variety of production rules rooted in ontological categories in order to generate a canonical conceptukl representation of the parsed sentence (cf. Section 6 ). This second level of interpretation is usually not taken into consideration by computational modcls of semantic interpretation, neither constituencybased nor depondency-based ones, although it, turus out to crucial for natumal language understanding.

\section{Grammar and Concept Knowledge}

Grammatical knowledge for syntactic analysis is based on a fully lexicalized dependency grammas: (Hahn et al., 1994). (our preference for dependency structures is motivated, among other things, by the observation that the correspondence of dependency relations (holding betwon lexical items) to conceptual relations (holding between the eoncepts they denote) is much closer than for constituency-based grammars (Hajicova, 1987). Hence, a dependencybased approach eases inherently the description of the regulatitios underlying semantic interpotation.

In this lexicalized dependency fannework. lexeme specifications form tho leaf nodes of a lexicon DAG, which are further abstracted in terms of lexome class specifications at diflorent levels of generality (cf. Figure 1), This leads to a lexome class hicrarchy, which consists of lexeme class names $\mathcal{W}:=$ \{VERISAL, VERIBINTRANS, NOMINAL, NOUN, ... $\}$ and a subsumption rolation $i s a_{\mathcal{W}}=\{$ (VERIBNTRANS,

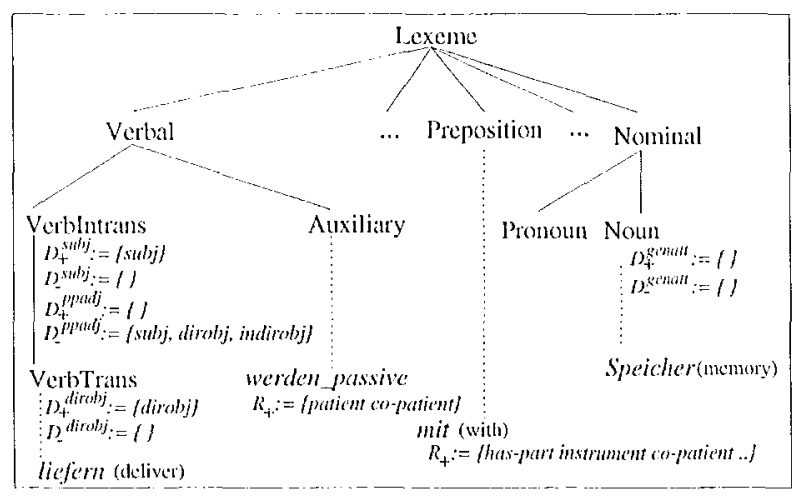

Figure 1: Fragment of the Jexome Class Hicrarchy 


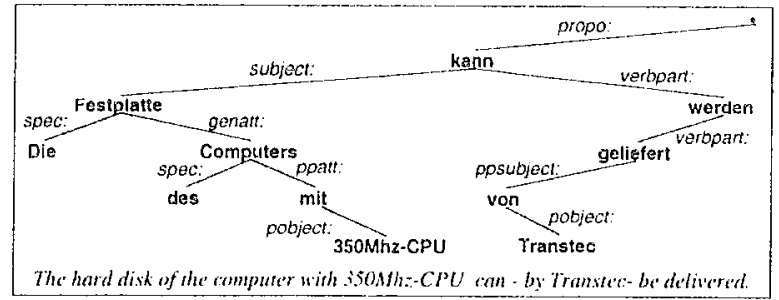

Figure 2: A Sample Dependency Graph

VERBAL $),($ NOLN, NOMNAL),$\ldots\} \subset \mathcal{W} \times \mathcal{W}$. Inheritance of grammar knowledge is based on the idea that constraints are attached to the most general lexeme classes to which they apply, leaving room for more and more specific (possibly, even idiosyncratic) specifications when one descends this hierarchy.

A dependency grammar captures binary constraints between a syntactic head (e.g., a noun) and one of its possible modifiers (e.g., a determiner or an adjective). In order to establish a dependency relation $\delta \in \mathcal{D}:=\{$ specifier, subject, dirobject, ... betweon a head and a modifier, lexeme-class-specific constraints on word order, compatibility of morphosyntactic features and semantic integrity must be fulfilled. Figure 2 depicts a dependency graph in which word nodes are given in bold face and dependency relations are indicated by labeled edges.

Conceptual knowledge of the underlying domain is expressed in terms of a KL-ONE-like knowledge representation language (Woods and Schmolze, 1992). The domain ontology consists of a set of concept names $\mathcal{F}:=\{$ COMPANY, HARIJ-DISK, ... $\}$ and a subsumption relation $i s a_{\mathcal{F}}=\{$ (HARD-DISK, STORAGEDevice), (Transtlec, Company), ... $\} \subset \mathcal{F} \times$ $\mathcal{F}$. The set of relation names $\mathcal{R}:=$ IIAS-PART, DELIVER-AGENT, ... denotes conceptual relations which are also organized in a subsumption hierarchy $i s a_{\mathcal{R}}=$ ( (HAS-HARD-DISK, HAS-PHYSICAL-PART), (IIAS-PHYSICAL-PART, HAS-PART) ,... $\}^{1}$ Examples of emerging concept and relation hierarchies are depicted in Figure 3 (right box).

In our approach, the representation languages for semantics and domain knowledge coincide (for arguments supporting this view, cf. Allen (1993)). Linking lexical items and conceptual entities proceeds as follows: Upon entering the parsing process, each lexical item $w$ that has a conceptual correlate $C$ in the domain knowledge base, $w . C \in \mathcal{F}$ (mostly verbs, nouns and adjectives), gets immediately instantiated in the knowledge base, such that for any instance $I_{w}$, initially, ${ }^{2}$ type $\left(\mathrm{I}_{w}\right)=w . C$ holds (e.g., $w=$ "Festplatte", $\mathrm{I}_{w}=$ HARD-DISK.2, w.C = type (HARDDISK.2) = HARD-DISK). If several conceptual correlates exist, either due to homonymy or polysemy,

\footnotetext{
${ }^{1}$ All subsumption relations, isa $\mathcal{W}$, is $a y$, and isa, are considered to be transitive and reflexive.

${ }^{2}$ For instance, anaphora might, necessitate changes of this initial reference assignment, cf. Strube and Hahn (1999).
}

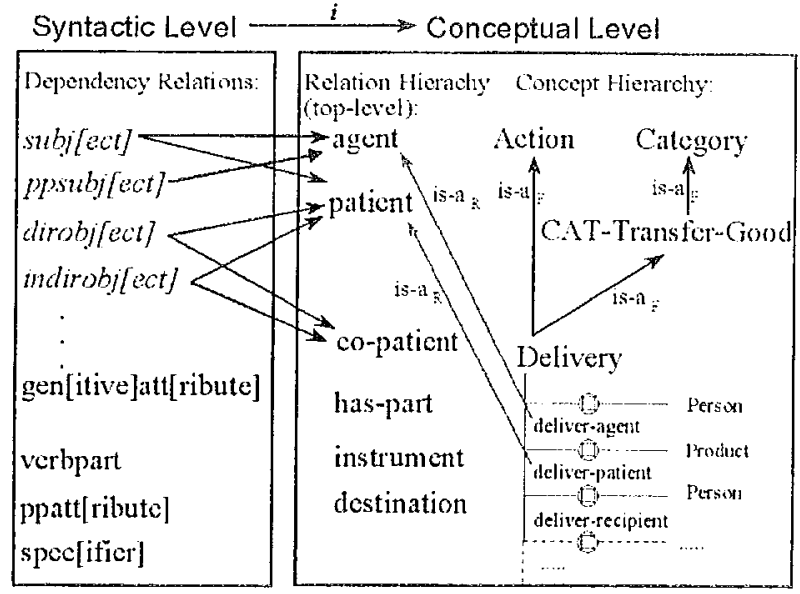

Figure 3: Relating Grammatical (left box) and Conceptual Knowledge (right box)

each lexical ambiguity is processed independently within separate context partitions of the knowledge base (Romacker and Hahn, 2000a).

\section{Interpretable Subgraphs}

In the parse tree from Figure 2, wo can distinguish lexical nodes that have a conceptual correlate (e.g., "Festplatte" relating to HARD-Disk, "geliefert" relating to Deluvery) from others that do not have such a correlate (e.g., "mit" (urith), "von" (by)). Semantic interpretation capitalizes on this distinction in order to find adequate conceptual relations between the corresponding concept instances:

Direct Linkage. If two word nodes with conceptual correlates are linked by a single dependency relation, a direct linkage is given. Such a subgraph can immediately be interpreted in terms of a conceptual relation licensed by the corresponding dependency relation. This is illustrated in Figure 2 by the direct linkage between "Festplatte" (hard disk) and "Computers" via the gen[itive]att[ribute] relation, which gets mapped to the HARD-DISK-OF role linking the corresponding conceptual correlates, viz. HARD-DISK.2 and COMPUTER-SYSTEM.4, respectively (see Figure 4). This interpretation uses only knowledge about the conceptual correlates and the linking dependency relation.

Indirect Linkage. If two word nodes with conceptual correlates are linked via a series of depen-

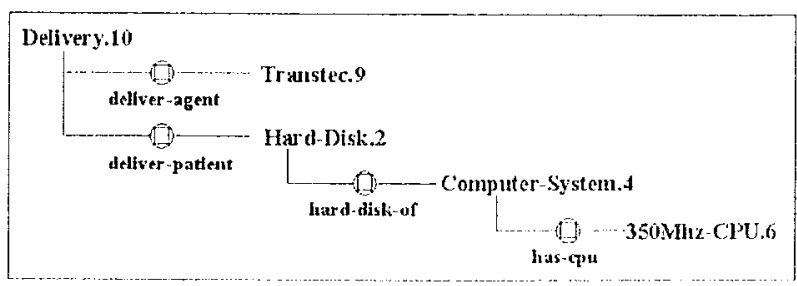

Figure 4: Semantic Interpretation of the Dependency Graph from Figure 2 
dency relations and none of the intervening nodes have a conceptual correlate, an indirect linkage is given. For such a "minimal" subgraph, semantic interpretation is made dependent on lexical information from the intervening nodes, as well as knowledge abont the conceptual correlates and dependency relations. Figure 2 illustrates such a configuration by the linkage between "Computers" and " $350 \mathrm{Mhz}$ $C P U$ " via the intervening node "mit" (with) and the ppatt[ribute] and pobject relations, the result of which is a conceptual linkage betweon Comp UT'ERSystram.4 and 350Mnz-CPU.6 via the relation IIASClPU in Figure 4.

Th order to ineroase the generality and to preserve the simplicity of semantic interpretation we introduce a genemalization of the notion of dependency relation such that it incorporates direct as well as indirect linkage: Two content words (nouns, adjectives, adverbs or full verbs) stand in a mediated syntactic relation, il one can pass from one word to the other along the connecting edges of the dependency graph without traversing word nodes other than prepositions, modal or anxiliary vorbs (i.c., elements of closed word classes). In Figure 2, e.g., the tuples ("Festplatte", "Computers") or ("Computers", "350Mhz-CPU") stand in mediated syntalctic relations, whereas, e.g., the tuplo ("Festplatte", "Transter") does not, since the connecting path contains "geliefert" (delivered), a content word.

This loads to the following definition: Let $w$ and $w^{\prime}$ be two content words in a sentence $S$. In addition, let $w_{2}, \ldots, w_{n-1} \in S(n \geq 2)$ bo prepositions, auxiliary or modal vorbs, and $w_{1}:=w$ and $w_{n}:=$ $w^{\prime}$. I'luen wo say that $w$ and $w^{\prime}$ stand in a mediated syntactic relation, ifl there exists an index $l \in\{1$, $\ldots, 11\}$ so that the following two conclitions hold:

1. $w_{i}$ is modifier of $w_{i-1}$ for $i \in\{1, \ldots, l-1\}$;

2. $w_{i}$ is head of $w_{i+1}$ for $i \in\{l, \ldots, 11-1\}$.

Wo call a subgraph identilied by such a series $w_{1}$, $\ldots, w_{n}$ a semantically interpretable subgraph of the dependency graph of $S$. The definition of a modiated syntactic relation encompasses the notion of a direct linkage $(n:=2$, so that an empty set of intervening nodes emorges). The special cases $l:=1$ and $l:=n$ yicld an asconding and descending scries of head-modificr relations, respectively.

\section{Semantic Interpretation Model}

The model of semantic interpretation we propose comprises two constraint layers. First, static constraints for somantic interpretation derived from directly mapping dependency relations to conceptual roles, and, second, a search of the knowledge base which dynamically takes these static constraints into account. The translation from the syntactic to the semantic level is achieved in a strictly compositional way by incrementally combining the conceptual representiations of semantically interpretable subgraphs 1until the entire dependency graph is processed.

Static Constraints. Interpretation procedures operating on semantically interpretable subgraphs may inherit restrictions from the type of dependency relations or from the lexical material they incorporate. Constraint knowledge from the grammar level comes in two varieties, viz. via a positive list, $D_{+}^{\text {lexval, }}$, and a negative list, $D_{-}^{\text {lexval }}$, of dependency relations, from which admitted as well as excluded conceptual relations, $R_{\tau_{-}}$and $R_{-}$, respectively, are derived by a simple static symbol mapping.

Knowledge about $D_{+}^{\text {terual }}$ and $D_{-}^{\text {lexval }}$ is part of the valency specifications. It is encoded at the level of lexeme classes $\mathcal{W}$, such that lexval $\in \mathcal{W} \times \mathcal{D}$. By way of property inheritance this knowledge is passed on to all subsumed lexical classes and instances. For instance (cf. Figure 1), the lexeme class of intransitive verbs, VHRIBN'RANS $\in \mathcal{W}$, defines for its subject valency $D_{+}^{\langle\text {(verbintrans, subject }\rangle}:=\{$ sul ject $\}$ and $D_{-}\langle$verbintrans, subject $:=\emptyset$, whereas for preposi-

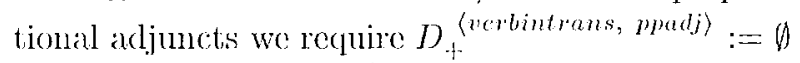
and $D_{-}^{\langle\text {ecrbintrans, pradj }\rangle}:=$ subjoct, dirobject, indirobject\}. All these constraints are inherited by the loxeme class VERB'lRANS. Wo then distinguish three basic cases how corresponding constraints may affect semantic interpretation processes:

1. Knowledge available from syntax determines the semantic interpretation, if $D_{+}^{\text {lex }}$ wal $\neq \emptyset$ and $D_{-}^{\text {lexul }}=\emptyset$ (e.g., the subject of a verb).

2. Knowledge available from syntax restricts the semantic: interprotation, if $D_{+}^{\text {lexeal }}=\emptyset$ and $D_{-}^{\text {lexwal }} \neq \emptyset$ (c.g., for prepositional adjuncts).

3. If $D_{+}^{\text {lexal }}=\emptyset$ and $D_{-}^{\text {lexval }}=\emptyset$, no syntactic constraints apply and semantic interpretation proceeds entarely concept-drinen, i.c., it relies on domain knowledge only (o.g., for genitives). ${ }^{3}$

In order to transfer syntactic constraints to the conceptual level, we define $i: \mathcal{D} \rightarrow 2^{R}$, a mapping from dependency relations onto sets of conceptual relations. Some of these mappings are already depicted in Figure 3 (e.g., $i$ (subject) $:=\{\Lambda$ GEN'T, PATIEN' $\}$ ). For dependency relations $\delta \in \mathcal{D}$ that cannot be linked a priori to a conceptual relation (o.g., gen[itive]att [ribnte]), we require $i(\delta):=0$.

The conceptial restrictions, $R_{+}$and $R_{-}$, must be

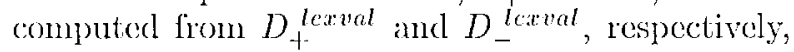
by applying the interpretation function $i$ to cach olcment of the corresponding sots. This leads us to $R_{+}:=\left\{y \mid x \in D_{+}^{\text {lexwal }} \wedge y \in i(x)\right\}$ and $R_{-}:=$ $\left\{y \mid x \in D_{-}^{l c x v a l} \wedge y \in i(x)\right\}$.

\footnotetext{
${ }^{3}$ We have currently no empirical evidence for the fourth possible case, where $D_{+}^{\text {leavel }} \neq 0$ and $D_{-}^{\text {lexval }} \neq 0$.
} 
Dynamic Constraint Processing. Scmantic interpretation implies a search in the knowledge base which takes the constraints into account that derive from a particular dependency parse tree. Two sorts of knowledge then have to be combined - first, a pair of concepts for which a connecting relation path has to be determined; second, conceptual constraints on permitted and excluded conceptual relations when connected relations are being computed. The first constraint type incorporates the content words linked by the semantically interpretable subgraph, the latter accounts for the particular dependency rolation(s) holding between them. Schema (1) describes the most general mapping from the conceptual correlates, h. $C_{\text {from }}$ and $m . C_{t o}$, in $\mathcal{F}$ of the two syntactically linked lexical items, $h$ and $m$, respectively, to connected relation paths $R_{c o n}$.

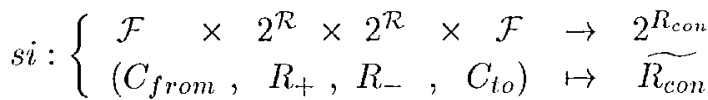

A connected relation path $r e l_{c o n} \in R_{c o n}$ is defined by:

$$
\begin{gathered}
\operatorname{rel}_{\text {con }}\left(\left(r_{1}, \ldots, r_{n}\right)\right): \Leftrightarrow \forall i \in\{1, \ldots, n-1\}: \\
i \operatorname{sa}_{\mathcal{F}}\left(\operatorname{type}\left(\operatorname{range}\left(r_{i}\right)\right), \operatorname{type}\left(\operatorname{domain}\left(r_{i+1}\right)\right)\right)
\end{gathered}
$$

A relation path is called connected, if for all its $n$ constituent, noncomposite relations $r_{i}$ the concept type of the domain of the relation $r_{i+1}$ subsumes the concept type of the range of the relation $r_{i}$.

To compute a semantic interpretation, si triggers a search through the knowledge base and identifics all connected relation paths from $C_{\text {from }}$ to $C_{t o}$. Due to potential conceptual ambiguitics in interpreting syntactic rolations, more than one such path may exist (hence, we map to the power set of $R_{\text {con }}$ ). In order to constrain connectivity, si takes into consideration all conceptual relations $R_{+} \subset \mathcal{R}$ a priori permitted for semantic interpretation, as well as all relations $R_{-} \subset \mathcal{R}$ a priori excluded. Both of them reflect the constraints set up by particular dependency relations or non-content words figuring as lexical relators of content words. Thus, rel $\in \widehat{R_{c o n}}$ holds, if $r e l$ is a connected relation path from $C_{f r o m}$ to $C_{t o}$, obeying the restrictions imposed by $R_{+}$and $R_{-}$.

If the function si returns the empty set (i.e., no valid interpretation can be computed), no dependency relation will be established. Otherwise, for all resulting relation paths $R_{\mathrm{AL}_{i}} \in \widetilde{R_{\text {con }}}$ an assertional axiom of the form $\left(h . C_{f r o m} \mathrm{REL}_{i} m . C_{t o}\right)$ is added to the knowledge base, where $\mathrm{REI}_{i}$ denotes the $i^{t h}$ reading. If $i>1$, conceptual ambiguities occur, resolution strategies for which are described in Romacker and Hahn (2000a).

To match a concept definition $C$ against the constraints imposed by $R_{+}$and $R_{-}$, we define the function get-roles $(C)=$ : $C R$, where $C R$ denotes the set of conceptual roles associated with $C$, which are then used as starting points for the path search. For ease and generality of specification, $R_{+}$and $R_{\text {- consist of }}$ the most general conceptual relations only. Hence, the concrete conceptual roles $C R$ and the general ones in $R_{+}$and $R_{-}$may not always be compatible. So prior to semantic interpretation, wo expand $R_{+}$ and $R_{-}$into their transitive closures, incorporating all their subrelations in the relation hierarchy. Thus, $R_{+}^{*}:=\left\{r^{*} \in \mathcal{R} \mid \exists r \in R_{+}: r^{*} i s a_{\mathcal{R}} r\right\} . R_{-}^{*}$ is correspondingly defined. $R_{+}$restricts the scarch to relations contained in $C R \cap R_{+}^{*}$, iff $R_{+}$is not cmpty (otherwise, all elements of $C R$ arc allowed), whereas

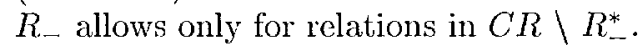

\section{A Sample Semantic Interpretation}

Whenever a scmantically interpretable subgraph is complete, scmantic interpretation gets started immediately. As an example, we will consider a case of indirect linkage, as illustrated by the occurrence of auxiliary and modal verbs within a passive clause.

When interpreting indirect syntactic relations, information not only about content word nodes but also about intervening noncontent word nodes becomes available. This way, further static constraints are imposed on $R_{+}$(and $R_{-}$) in terms of a list $R_{\text {lex }}$ $\subset \mathcal{R}$ of permitted conceptual relations. This information is always specified at the lexome level. Since $R_{l e x}$ relates to closed-class items only, the required number of specifications is casy to survey.

In our example (cf. Figure 2), the content words "Festplatte" (hard disk) and "geliefert" (delivered) are linked by a mediating modal verb ( "kann" (can)) and a passive auxiliary ("werden" (be passive)). The semantic interpretation schema for passive auxiliaries (2) addresses the concept type of the instance for their syntactic subject, $C_{s u b j}=t y p e\left(\mathrm{I}_{s u b j}\right)=$ HARD-DISK, and that for their verbpart, $C_{\text {verbpart }}$ $=\operatorname{type}\left(\mathbf{I}_{\text {vertpart }}\right)=$ DELIVERY. The relation between these two, however, is determined by $R_{\text {passaux }}$ $:=\{$ PATIENT, CO-PATIENT $\}$, constraint knowledge which resides in the lexeme specification for "werden" as passive auxiliary (cf. Figure 1).

$s i_{a u x}:\left(C_{\text {verbpart }}, R_{\text {passaux }}, \emptyset, C_{\text {sulj }}\right) \mapsto \widetilde{R_{c o n}}$

With $s i_{\text {aux }}$ (DELIVERY, \{PA'TEN'T, CO-PATIENT $\}$, (b) HARD-DisK), we get the conceptual relation DELIVER-PATIENT (cf. Figure 3), since HARD-Disk is subsumed by PronUCT and, thus, a legal filler of DELIVER-PATIENT $\in R_{\text {passaux }}^{*}$.

\section{Conceptual Interpretation}

Conceptual interpretation uses a production rule system (Yen et al., 1991) which accounts for characteristic patterns of assertions that result from the semantic interpretation process. While the outcome of semantic interpretation (cf. Figure 4) still adheres 
to the surface form of the parsed sentence, concep)tual interpretation abstracts away from these sinface phenomena and creates a 'normalized', canonical conceptual representation of the input, as neceled. c.g., for uniformly querying the knowledge base.

As an example of such inferences consider figure. 5, whth the DenVERS relation linking TRANSTEC.9, a hardware supplice, and HARD-I) IsK.2. By computing a conceptual relation representing the nndorlying ACTION TRANSTbG. and HARI)-DISk.2 are integrated in a normalized concept graph. Note that the corresponding lexical items, "rhanstec" and "Festplatte" (hard disk), are not linked via a mediated syntactic relation in Figure 2 . Hence, we may clearly discern semantic interpretation, which operates on single semantically interpretable subgraplis only, from conceptual interpretation, where the inference-based interpretation of relationships among different subgraphs comes into play.

An independent level for conceptual interpretation also became a necessity due to analytic considerations. Often the local constraints for conceptual roles of ACIION, STATE, or EVINT concepts camot be formulated restrictive onough for the semantic; interpretation process. For example, the conceptual correlate of the verb "possess" does not impose any restriction on its PITIENT role (linked to the subject dependency relation in a semantically interpretable subgraph). Rather, restrictions apply to properly relating the filler of the bATENT slot with that of the co-PATIEN slot (dirobject at the dependency lewel). Conceptual interprotation rules are a moans to further constrain these! 'context-sensitive' aspects of the interpretation process.

Since verbs play a prominent role in dependency grammars, the production mule system for conceptual interpretation is based upon the conceptual eorrelates of vorbs (henceforth verts concepts) in the? kuowledge base. I Different viows are clefined for verb concepts by using three abstraction dincensions.

First, vorb concepts are classified, according to the set of thematic roles they supply, as ACTION, STATE or PRocess. Delatem, og., is assigned to ACTHON, since both AGENT and PATENe form part of the concept definition (cf. Figure 3, right box).

The second level of abstraction consists of categorizations which reflect a common core meaning. "The upmost conceptual node in this hiorarchy is CATScory. DELIVIRY, e.g., is considered as a concept which represents the Acrion of transfering a $G(O) D$

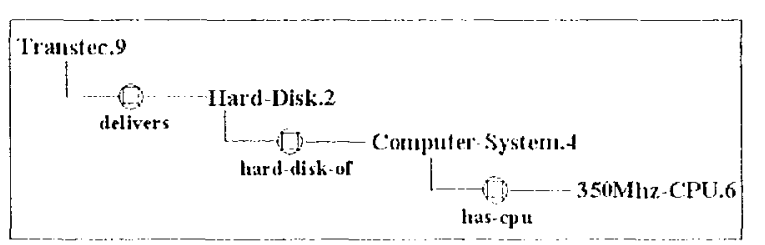

Figure 5: A Sample Conceptual Interpretation of the Dependency Graph from Figure 2 to a castomer. All verb concepts bolonging to this category ane subsumed by the corresponding concept CAT - Thansfir. GoOd). (We here make use of multiple inheritance mechanisms.)

Finally, every verb concept is linked to some Verb-MODEL. DERIVERY or any other verb concept. of the CAT-Transpre-Goon category is a comstituent phatse of the BWY-AND-SELL-NODFL. To generalize appropriately from individual verbs, verb catcogries were extracted from on text corpora that further rofine a large-scale taxonomy for German verbs (Balluter and Bromenstuhl, 1986). In this work, a total of about 20,000 verbs were subsumed by 700 categories to reflect a semantic generalization in terms of a hierarchy of verb categories.

The production rules for conceptual interpretation operate on this categorial hierarchy. Every verb concept in the hierarchy is a subconcept of exactly one category in the knowledgo base. Whenever the preconditions of an interpretation rule are fulfilled, a conceptual interpretation is computed.

Conceptual and semantic interpretation depend on each other, since the basic interpretation schema (cf. expression (1) in Section 4) is supplied with actual parameters from production rules. We therefore may define another specialization of the basic interpretation schema for conceptual interpretation sicone. In particular, path searches are triggered that are restricted by a positive list rendered by the applicable production rule.

For our sample sentence (cf. Figures 2 and 4 ), the conceptual correlate for the verb "delivers" (DFLIVERY) is a subconcept of ACrrox. Additionally, DEnERY is a subconcept of the category CAT-Transter-GoOn) (cf. Figure 3). The corresponding conceptual interpretation rule is given in Figure 6. Whenever an instance of the category CAT-TRANSFER-GOON is encountered and both its ACENT and PATIN' roles are filled, relation paths are computed from the types of the two instances involved, $a$ ancl $p$, respectively. For each relation found by the search algorithm (REL in Figure 6), a corresponding assertion is added to the knowledge base (T'ELL in Figure 6). In the example, the interpretation schema is instantiated with the 4-tuple (COMPANY, \{TRANSFRKS-GOOD $\},\{\}$, HARD-DLSK) resulting in the computation of \{1)eluvens\} as the proper rolation link (cf. Figure 5), since it is a subrelation of TRANSFERS-COOD.

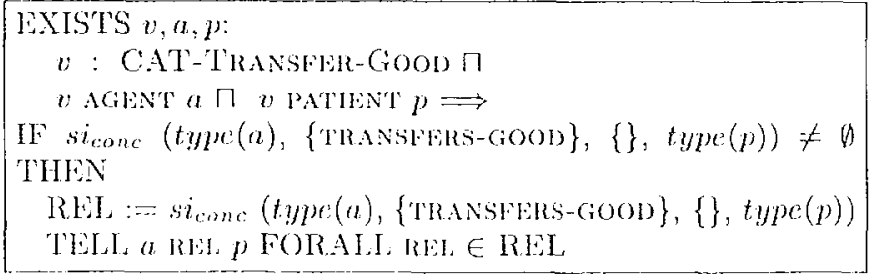

Figne 6: Sample Conceptual Interpotation Rule 


\section{Evaluation}

We evaluated this approach to semantic interpretation on a random selection of 54 texts (comprising 18,500 words) from two text corpora, viz. consumer product test reports and medical finding reports. For evaluation purposes, we concentrated on the interpretation of genitives (as an instance of direct linkage) and on the interpretation of periphrastic verbal complexes, i.e., passive, temporal and modal constructions (as instances of indiroct linkage).

The underlying ontology consists of an upper generic part (containing about 1,500 concepts and relations) and domain-specific extensions relating to information technology (IT) and (parts of) anatomical medicine (MED). Each of these two domain modcls adds about 1,400 concepts and relations to the upper model. Corresponding lexeme entries in the lexicon provide linkages to the cntirc ontology.

We considered a total of 247 genitives in the sample. Recall was higher for medical texts $(57 \%)$ than for IT documents (31\%), though, in general, rather low. However, precision peaked at $97 \%$ and $94 \%$ for medical and IT texts, respectively. The mumber of syntactic constructions with modal verbs or auxiliaries amout to 292 examples. Compared to genitives, we obtained a slightly more favorable recall for both domains - $66 \%$ for MED, $40 \%$ for IT while precision dropped slightly to $95 \%$ and $85 \%$ for medical and IT documents, respectively. ${ }^{4}$

As with any such cvaluation, idiosyncrasies of the coverage of the knowledge bases are inevitably tied with the results and, thus, put limits on too farreaching generalizations. However, our data reflect the intention to submit a knowledge-intensive text understander to a realistic, i.e., conceptually unconstrained and therefore "unfriendly" tost environment. Judged from the figures of our recall data, there is no doubt, whatsoever, that conceptual coverage of the domain constitutes the bottleneck for any knowledge-based approach to NLP." Sublanguage differences are also mirrored systematically in these data, since medical texts adhere more closely to well-established concept taxonomies and writing standards than magazine articles in the IT domain, whose rhetorical styles vary to a larger degree.

\section{Related Work}

The standard way of deriving a semantic interpretation for constituency-based grammars is to assign each syntactic rule one or more semantic interpretation rules (e.g., van Eijck and Moore (1992)), and to

\footnotetext{
${ }^{4}$ A more detailed presentation of this evaluation study is given in Romacker and Hahn (2000b).

${ }^{5}$ For the medical domain at least, we are currently actively pursuing research on the semiantomatic creation of large-scale ontologies from weak knowledge sources, viz. medical terminologies; cf. Schulz and Hahn (2000).
}

determine the meaning of the syntactic head from its constituents. This approach has also been adopted in the few explicit attempts at incorporating semantic interpretation into a dependency grammar framework (Milward, 1992; Lombardo et al., 1998). There are no constraints on how to design and organize this rule set despite those that are implied by the choice of the semantic theory. In particular, abstraction mechanisms (going beyond the level of sortal taxonomies for semantic labels, cf., c.g., Creary and Pollard (1985)), such as property inheritance, defaults, are lacking. Accordingly, the number of rules increases rapidly and easily reaches orders of sevcral hundreds in a real-world setting (Bean ot al., 1998). As an alternative, we provide a small set of generic semantic interpretation schemata (by the order of 10) and conceptual interpretation rules (by the order of 30 for 200 verb concepts) instead of assigning specific interpretation rules to each grammar item (in our case, single lexemes), and incorporate inheritance-based abstraction in the use of these schemata during the interpretation process in the knowledge base. We clearly want to point out that while this rule system covers a wide varicty of standard syntactic constructions (such as genitives, prepositional phrases, various tense and modal forms), it currently does not account for quantificational issues (like scope ambiguities) for which entircly logic-based approach (Charniak and Goldman, 1988; Moore, 1989; Pereira and Pollack, 1991) provide quite sophisticated solutions.

Sondheimcr et al. (1984) and Hirst (1988) treat semantic interpretation as a direct mapping from syntactic to conceptual representations. They also share with us the representation of domain knowledge using KL-ONE-style terminological languages, and, hence, they make heavy use of property inheritance (or typing) mechanisms. The main difference to our alproach lies in the status of the scmantic rules. Sondheimer et al. (1984) attach single intorpretation rules to each role (filler) and, honce, have to provide utterly detailed specifications reflecting the idiosyncrasies of each semantically rolevant (role) attachment. Property inhoritance comes only into play when the selection of alternative semantic rules is constrained to the onc(s) inherited from the most specific case frame. In a similar way, Hirst (1988) uses strong typing at the conceptual object level only, while we use it simultaneously at the grammar and the domain knowledge level for the processing of semantic schemata.

\section{Conclusions}

We introduced an approach to the design of compact, yet highly expressive semantic interpretation schemata. They derive their power from two sources. First, the organization of grammar and domain 
knowledge, as well as semantic interpretation mechanisms, are based on inheritance principles. Second, interpretation schemata abstiact from particular linguistic phenomena (specific loxical itens, lexeme classes or dependency relations) in terms of general configuration patterns in dependency graphs.

Underlying these design decisions is a strict separation of linguistic and conceptual knowledge. A clearly defined interface is provided which allows these specifications to make reference to fine-grained hierarchical knowledge, no matter whether it is of grammatical or conceptual origin. The interface is divided into two levels. One makes use of static, high-level constinaints supplied by the mapping of syntactic to conceptual roles or supplied as tho meaning of closed word classes. The other uses these constraints in a dynamic scarch through a knowledge base, that is parametrized by fow and simple schomati. Finally, at the lovel of conceptual interpretation inferences emerging from semantic representations are computed by a set of productions which make reference to a verbcategorial hicrarchy.

Also since the number of schematid at the semantic description layer remains rather small, their execution is casy to trace and thus supports the maintenance of large-scale NJP systems. The high abstraction level provided by inheritance-based somantic specifications allows casy porting across clifferent application clomains. Our experience rests on reusing the set of semantic selematiance developed for the information technology domain in the nedical clomain without further changes.

Acknowledgments. We want to thank the members of the $\overline{6 V}$ group for close cooperation. M. Romacker was supported by a grant from DFG (Ha 2097/5-1).

\section{References}

J. Allen. 1993. Natural language, knowledge representation, and logical form. In M. Bates and R. Weischedel, editors, Challenges in Natural Language Processing, pages $146-175$. Cambridge University Press.

T. Ballmer and W. Brennonstuhl. 1986. Deutsche Verben. Hine sprachanalytische Untersuchung des deutschen Verbwortschatzes. Tübingen: G. Narr.

C. Bean, T. Rindílesch, and C. Snciderman. 1998. Automatic semantic interpretation of anatomic spatial relationships in clinical text. In Proc. 1998 AMIA Annual Fall Symposium, pages 897-901.

E. Charniak and R. Goldman. 1988. A logic for semantic interpretation. In Proc. of the "6th Annual Meeting of the $A C I$, pages 87-94.

L. Creary and C. Pollard. 1985. A computational semantics for natural language. In Proce of the 23rd Annual Mecting of the ACI, parges 172-179.

M. Dalrymple. 1992. Categorial scmantics for LFG. In COLING'92 - Proceedings of the 15th [sic! 14th] International Conference, jages 21.2-218.
U. Hahn, S. Schacht, and N. Bröker. 1994. Concurrent, object-oriented natual language parsing: the PARST TALK model. International Journal of IIuman-Computer Studies, 41(1/2):179-222.

E. Hajicova. 1987. Linguistic meaning as rolated to syntiax and to scmantic interpretation. In M. Nagao, editor, Language and Artificial Intelligence, pages 327-351. North-Holland.

G. Hirst. 1988. Scmantic interpretation and ambiguity. Artificial Intelligence, 34(2):131-177.

V. Lombardo, L. Losmo, L. Ferraris, and C. Seidenari. 1998. Incremental interpretation and lexicalized grammar. In CogSci'98 - Proceedings of the 20th Annual Conference, pages 621-626.

D. Milward. 1992. Dynamics, dependency grammat and incremental interpretation. In COLING'92 Proceedings of the 15th [sic! 14th] International Conference, pages 1095-1099.

R. Moore. 1989. Unification-based semantic interpretation. In Procedings of the 27th Annual Mecting of the ACI, pages 33-41.

F. Poreira and M. Pollack. 1991. Incremcntal interpretation. Artificial Intelligence, 50(1):37-82.

M. Romalder and U. Hahn. 2000a. Coping with difforent types of ambiguity using a uniform context handling mechanism. In Applications of Natural Language to Information Systems. Proceedings of the 5 th NLDB Conference.

M. Romacker and U. Haln. 20001). An cmpirical assessment of semantic interpretation. In Proc. of the 6th Applied Natural Language Processing Conference 18 st Conference of the North American Chapter of the ACL, pages 327-334.

S. Schulz and U. Iahn. 2000. Knowledge engineesing by lange-scale knowledge reuse: experience from the medical domain. In $K R$ 'gogo - Proe. $7 t h$ International Conference, pages 601-610.

N. Sondheimer, R. Weischedel, and R. Bobrow. 1.984. Scmantic interpretation using KL-ONL. In COLING'84 - Proc. 10th Inl. Conference \& gend Annual Mecting of the ACL, pages 101-107.

M. Strube and U. Hahn. 1999. Functional centering: grounding referential coherence in information structure. Computational Linguistics, 25(3):309344.

J. van Jijck and R. Moore. 1992. Scmantic rules for English. In H. Alshawi, editor, The Core Language Engine, pages 83-115. MIT Press.

J. Wedekind and R. Kaplan. 1993. [Type-driven semantic interpretation of f-structures $\langle<J, W\rangle$ $,<R, K>>$ ]. In EACL'93 - Proc. 6th Conf. European Chapter of the $A C L$, pages 404-411.

W. Woods and J. Schnolzc. 1992. The KI-ONIs family. Computers 8 Mathematics with Applications, $23(2 / 5): 133-177$.

J. Yen, R. Neches, and R. MacGregor. 1991. CLASP: integrating torm subsumption systems and production systems. IEEE Transactions on Knouledge and Data Engineering, 3(1):25-32. 